\title{
Cáncer de cabeza y cuello en Uruguay. Análisis de sobrevida en dos centros de referencia
}

\author{
Diego Álvarez Arias*, Andrés Munyo Estefan+, ${ }^{\dagger,}$, German Borche ${ }^{\dagger, \neq}$, \\ Ricardo D’Albora§, Virginia Rodríguez Sande`, Mauricio Cuello**
}

\section{Resumen}

Introducción: el carcinoma escamoso de cabeza y cuello (HNSCC) es el cáncer de mayor prevalencia en la otorrinolaringología, ocupando en el Uruguay el quinto lugar en incidencia y el sexto lugar en mortalidad en hombres. El propósito de este estudio fue analizar la sobrevida global de pacientes con cáncer de cabeza y cuello en dos centros de referencia de Montevideo.

Método: se analizó de forma retrospectiva la sobrevida global de 436 pacientes diagnosticados con HNSCC entre los años 2005 y 2015. Se analizó la sobrevida por método de Kaplan-Meier en relación con el estadio tumoral, topografía, estatus de tabaquismo y alcoholismo de los pacientes. Para el estudio de la interacción de las variables con posible importancia en el pronóstico se utilizó el modelo de regresión de Cox.

Resultados: la mediana de sobrevida global de los pacientes con cáncer de cabeza y cuello en nuestra serie es de 35,8 meses (23,5-48,1, IC 95\%), para el cáncer de laringe fue superior a la del resto de los pacientes con cáncer de otros sitios anatómicos, $77,3(49,3-105,2$, IC 95\%) versus 26,2 meses (20,7-48,1, IC 95\%, p < 0,001). No hubo diferencia entre la sobrevida global de tabaquistas y no tabaquistas. El grupo de los no alcoholistas presentó una mejor sobrevida global que el grupo de los alcoholistas, 72,4 (39,1-105,7, IC 95\%) versus 26,7 (19,9-33,6 IC 95\%). El análisis multivariante identificó el estadio y el alcoholismo como factores pronósticos independientes en nuestra serie.

Conclusiones: la mediana de sobrevida global de nuestra serie es similar a la reportada en la bibliografía internacional. Los pacientes con cáncer de laringe presentan mayor sobrevida que los pacientes con cáncer de otros sitios otorrinolaringológicos. De los factores de riesgo clásicos, en nuestra serie el consumo de alcohol impacta negativamente en sobrevida de los pacientes con cáncer de cabeza y cuello.

Palabras clave: NEOPLASIAS DE CABEZA Y CUELLO

TABAQUISMO

ALCOHOLISMO

SOBREVIDA

URUGUAY
Key words: $\quad$ HEAD AND NECK NEOPLASMS

TOBACCO USE DISORDER

ALCOHOLISM

SURVIVORSHIP

URUGUAY

\footnotetext{
* Cátedra de Otorrinolaringología, Hospital de Clínicas, Universidad de la República. Montevideo, Uruguay.

† Asistente de Cátedra de Otorrinolaringología, Hospital de Clínicas, Universidad de la República. Montevideo, Uruguay.

‡ Servicio de Otorrinolaringología, Hospital Central de las Fuerzas Armadas, Ministerio de Defensa. Uruguay.

$\S$ Profesor Agregado de Otorrinolaringología, Hospital de Clínicas, Universidad de la República. Montevideo, Uruguay.

TI Residente de Oncología Médica, Hospital de Clínicas, Universidad de la República. Montevideo, Uruguay.

** Profesor Adjunto de Oncología Médica, Hospital de Clínicas, Universidad de la República. Montevideo, Uruguay.

Correspondencia: Diego Álvarez Arias. Dirección: Canelones 2398 apto 601. Correo electrónico: drdiegoalvarezarias@gmail.com

Los autores declaran no tener conflictos de interés en el desarrollo o publicación de este manuscrito.

Recibido: 29/9/17

Aceptado: $28 / 12 / 17$
} 


\section{Introducción}

En el año 2012 se estimaron mundialmente un total de casi 700.000 nuevos casos de cáncer de cabeza y cuello, con 6,5\% en América del Sur y América Central. Mundialmente el cáncer de cabeza y cuello se ubica en el séptimo lugar en mortalidad, representando aproximadamente unas 375.000 muertes anuales, de las cuales el $5,8 \%(21.762)$ ocurrieron en Latinoamérica ${ }^{(1)}$.

Estas regiones se caracterizan por una alta incidencia de cáncer de cabeza y cuello ${ }^{(2)}$; sin embargo, las tasas entre países varían ampliamente. Existe una disparidad de sexo en el cáncer de cabeza y cuello, reportándose en hombres $72 \%$ de los casos de cáncer de esta topografía en la región.

En Uruguay el cáncer de cabeza y cuello se ubica en el quinto lugar en incidencia y sexto en mortalidad en hombres ${ }^{(3)}$, ubicándose entre los primeros países en incidencia y mortalidad de América Latina ${ }^{(4)}$.

Son escasos los reportes sobre sobrevida del cáncer de cabeza y cuello en Latinoamérica, no existiendo comunicaciones recientes en nuestro país que hagan referencia a este punto. El objetivo de este artículo es analizar la sobrevida global de pacientes con cáncer de cabeza y cuello en dos centros oncológicos de referencia en Montevideo, Uruguay.

\section{Material y método}

Se realizó un estudio retrospectivo utilizando una base de datos de 436 pacientes asistidos en forma secuencial entre el 1 de enero 2005 y el 31 de diciembre de 2015 en las policlínicas oncológicas del Departamento de Otorrinolaringología del Hospital de Clínicas y del Servicio de ORL del Hospital Central de las Fuerzas Armadas. Se realizó un seguimiento periódico de los pacientes desde la fecha del diagnóstico hasta la fecha de cierre del estudio. Fue aprobado por los comités de ética de ambas instituciones en el marco del Estudio del cáncer de cabeza y cuello en Latinoamérica en colaboración con la IARC (estudio interCHANGE).

Las fechas de muerte fueron tomadas de la base de datos de la Comisión Honoraria de Lucha contra el Cáncer y de los registros médicos de ambos centros hospitalarios. Se tomaron datos de la población en estudio como sexo, edad, sitio anatómico de la lesión, fecha de diagnóstico para la cual se utilizó la fecha de confirmación anatomopatológica y el estadio tumoral al diagnóstico de acuerdo con los criterios de la AJCC.

Se analizó la prevalencia del consumo de alcohol y tabaco en la población al momento del diagnóstico utilizando los mismos criterios establecidos para el estudio interCHANGE de la IARC. Se definió como tabaquista a aquel paciente que hubiese consumido tabaco diaria- mente por lo menos durante un año y alcoholista a aquel paciente que hubiese ingerido por lo menos un trago de alcohol (20 mg de alcohol puro) por día en los últimos 30 días.

Se incluyeron a todos los pacientes con diagnóstico anatomopatológico de carcinoma epidermoide, excluyéndose pacientes con diagnóstico de otras variedades histológicas.

Se calculó la mediana de sobrevida global para toda la población y en función del sitio anatómico de la lesión (laringe versus no laringe), estadio TNM, condición de alcoholista/no alcoholista y tabaquista/no tabaquista por método de Kaplan-Meier, utilizando el test de Log Rank para comparar los distintos grupos. Los datos fueron procesados utilizando el software SPSS 19.0. Para el análisis de sobrevida y de las variables con posible importancia en el pronóstico se utilizó el modelo de regresión de Cox.

\section{Resultados}

Las características de la población se expresan resumidos en las (tabla 1). La mediana de edad fue de 62 años (rango: 40 - 84 años). La tabla 2 resume la distribución por topografía.

\section{Sobrevida y estadio tumoral}

La mediana de sobrevida global para todas las topografías y estadios fue de 35,8 meses $(23,5-48,1$, IC del 95\%).

Se agrupó a los pacientes en dos grupos, estadio temprano (estadios I y II de la AJCC) con 90 (20,7 \%) pacientes y estadio tardío (estadio III y IV de la AJCC) con $346(79,3 \%)$. La mediana de sobrevida para los estadios tempranos no se alcanza, mientras que para los estadios tardíos fue de 26,7 meses (19,6-33-7, IC 95\%). La sobrevida a cinco años para el grupo de estadios tempranos fue de $63,8 \%(53,6-74,0$, IC 95\%), mientras que para el grupo tardío fue de $32 \%(26,9-37,1$, IC $95 \%, p<0,005)$.

\section{Sobrevida del cáncer de laringe versus otros sitios}

La sobrevida global de los pacientes con cáncer de laringe se diferenció de la del resto de los sitios 77,3 (49,3-105,2, IC 95\%) versus 26,2 meses (20,7-48,1, IC $95 \%, \mathrm{p}<0,001$ ) (figura 2). La sobrevida a inco años para el cáncer de laringe fue de 54,3\% (46,8-61,8, IC $95 \%)$.

\section{Sobrevida y factores de riesgo}

En nuestro estudio $352(83 \%)$ pacientes eran fumadores, 297 (68\%) eran alcoholistas y 281 (64\%) combinaban ambos factores. La sobrevida global a cinco años de los pacientes tabaquistas fue de $38,9 \%$ (33,9-43,9, IC $95 \%)$ y de $45,3 \%(33,9-56,7$, IC $95 \%)$ para los no taba- 
Tabla 1. Características generales de la población

\begin{tabular}{lcccc}
\hline & $N=436(\%)$ & $\begin{array}{c}\text { Laringe } \\
n=169\end{array}$ & $\begin{array}{c}\text { Cavidad oral } \\
n=73\end{array}$ & $\begin{array}{c}\text { Orofaringe } \\
n=88\end{array}$ \\
\hline Edad (años) & 62 & & 52 & 76 \\
\hline Hombres & 352 & 150 & & $76(86,4 \%)$ \\
Mujeres & 84 & & $60(82,2 \%)$ & $73(82 \%)$ \\
\hline Tabaquismo & $362(83 \%)$ & $153(90,5 \%)$ & $50(68,4 \%)$ & 11 \\
\hline Alcoholismo & $297(68 \%)$ & $124(73,3 \%)$ & 17 & 74 \\
\hline Estadio TNM & & 53 & \\
\multicolumn{1}{c}{ Temprano (I y II) } & $20,7 \%$ & 40 & & \\
\hline Tardío (III y IV) & $79,3 \%$ & 116 & & 74 \\
\hline
\end{tabular}

Tabla 2. Distribución por sitio anatómico afectado

\begin{tabular}{lcc|}
\hline & $n$ & $\%$ \\
\hline Laringe & 169 & 38,7 \\
Glotis & 94 & \\
Supraglotis & 50 & \\
Subglotis & 2 & \\
Sin especificar & 22 & \\
Orofaringe & 88 & 20,2 \\
Cavidad oral & 73 & 16,7 \\
Hipofaringe & 35 & 8 \\
Ganglios linfáticos & 25 & 5,7 \\
Pirámide nasal, fosas nasales, & 20 & 4,9 \\
senos paranasales & & \\
Cavum & 11 & 2,5 \\
Parótida & 10 & 2,3 \\
Oído & 4 & 0,9 \\
Cara (párpado inferior) & 1 & 0,2 \\
Total & 436 & \\
\hline & & \\
\hline
\end{tabular}

quistas ( $p=0,964)$ (figura 3 ). En el caso de los pacientes alcoholistas la sobrevida a cinco años para fue de 52,2\% (43,9-60,5, IC 95\%) frente a $34,5 \%(29,1-39,9$, IC 95\%) para el grupo de alcoholistas $(\mathrm{p}=0,005)$ (figura 4).

Los resultados del análisis multivariable se resumen en la tabla 3.

\begin{tabular}{|c|c|c|c|}
\hline & $H R$ & IC 95\% & $p$ \\
\hline Edad & 1.015 & $1.003-1.027$ & 0.011 \\
\hline Sexo & 1.265 & $0.808-1.980$ & 0.304 \\
\hline $\begin{array}{l}\text { Estadio en } 2 \text { grupos } \\
\text { (temprano y tardío) }\end{array}$ & 2.376 & $1.556-3.629$ & 0.001 \\
\hline Tabaco & 0.616 & $0.342-1.109$ & 0.106 \\
\hline Alcohol & 1.476 & $1.027-1.922$ & 0.022 \\
\hline Tabaco + alcohol & 1.219 & $0.484-3.067$ & 0.674 \\
\hline
\end{tabular}

\section{Discusión}

Este es el primer estudio en analizar la sobrevida global de pacientes con cáncer de cabeza y cuello en Uruguay.

En nuestra serie el cáncer de laringe es el cáncer de cabeza y cuello de mayor prevalencia, incluso superando en números absolutos al cáncer de cavidad oral y orofaringe tomados en conjunto, sitios que predominan en la población general uruguaya ${ }^{(3)}$.

El cáncer de laringe está íntimamente ligado al consumo de tabaco y principalmente en el sector glótico ${ }^{(5)}$, el cual está altamente representado en nuestra casuística.

En el año 2009, el 25\% de la población uruguaya mayor de 15 años era fumadora ${ }^{(6)}$. La prevalencia asciende a $27,3 \%$ en hombres y hasta $33,1 \%$ para los niveles socioeconómicos más bajos ${ }^{(7)}$. El porcentaje de tabaquistas en la población en estudio es alto, $83 \%$, más aún en la población diagnosticada de cáncer de laringe con 90,5\%.

En nuestra casuística hay un franco predominio de los estadios avanzados, que como sabemos es el princi- 


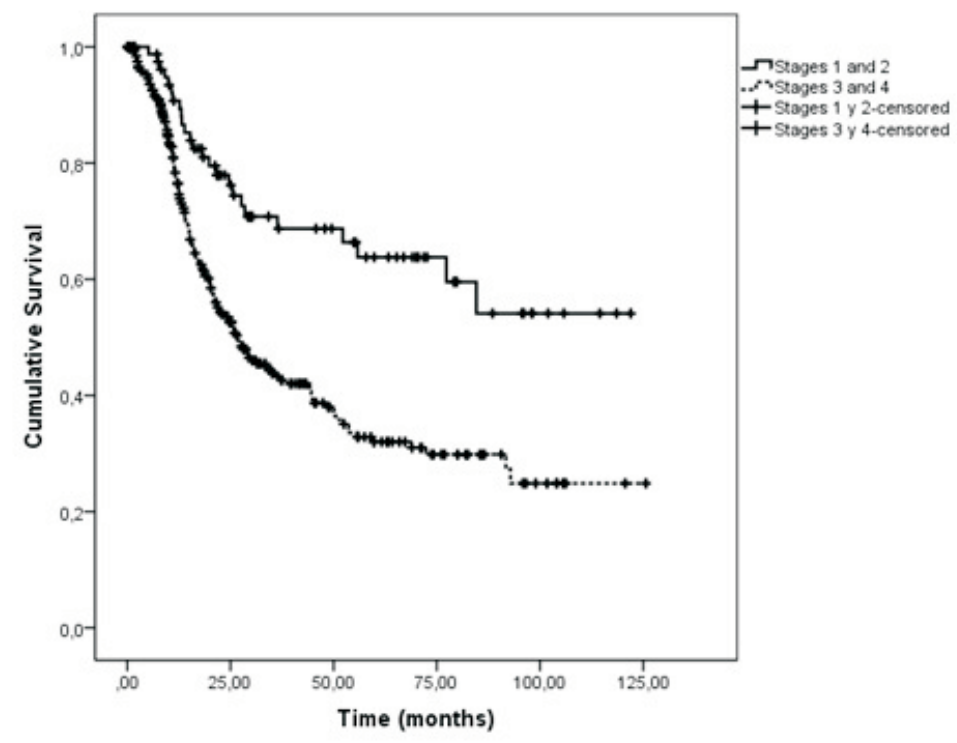

Figura 1. Funciones de sobrevida Kaplan-Meier por grupo según estadio TNM.

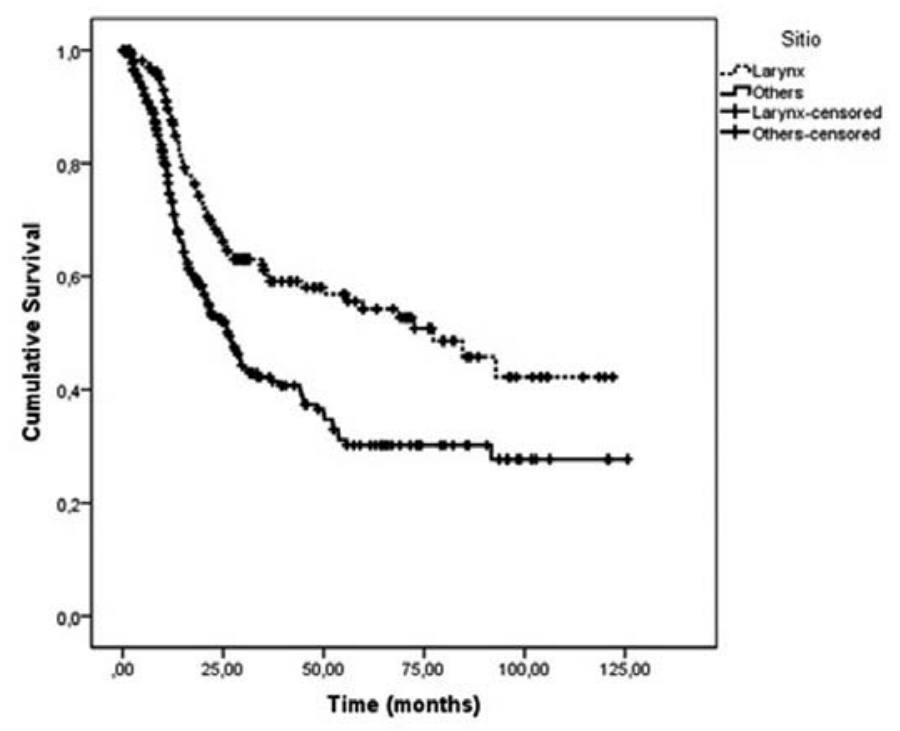

Figura 2. Funciones de sobrevida según sitio anatómico del cáncer de cabeza y cuello. Laringe versus no laringe.

pal factor pronóstico y la supervivencia en función de los estadios fue acorde a la bibliografía internacional considerando todos los sitios anatómicos ${ }^{(8,9)}$, debiendo destacarse que para un mismo estadio la sobrevida del cáncer de cabeza y cuello es muy variable según la topografía. La sobrevida global a cinco años para los estadios tempranos (I y II) varía de $80 \%$ para orofaringe y laringe a $45 \%$ para hipofaringe. En estadios tardíos (III y
IV) la sobrevida se encuentra entre $50 \%$ para orofaringe y $25 \%$ para hipofaringe ${ }^{(8,10)}$.

En nuestra serie la sobrevida del cáncer de laringe fue estadísticamente superior cuando se comparó con los otros sitios agrupados, este resultado puede ser consecuencia del alto porcentaje de pacientes con tumores glóticos. El plano glótico presenta un escaso drenaje linfático, lo que determina que el porcentaje de metástasis 


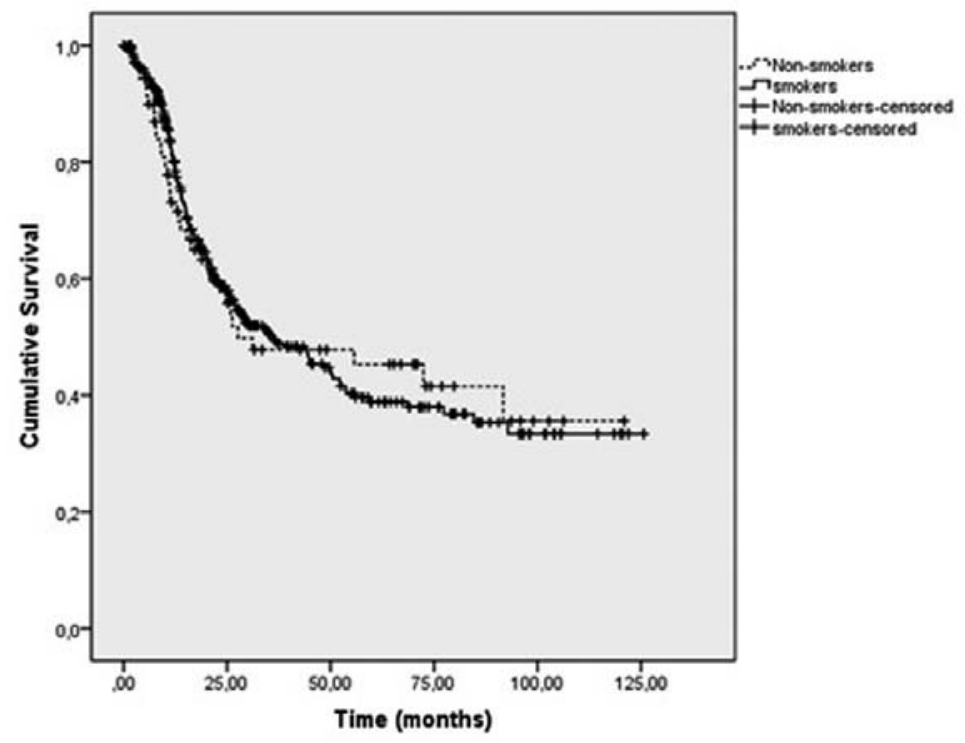

Figura 3. Funciones de sobrevida de pacientes tabaquistas y no tabaquistas.

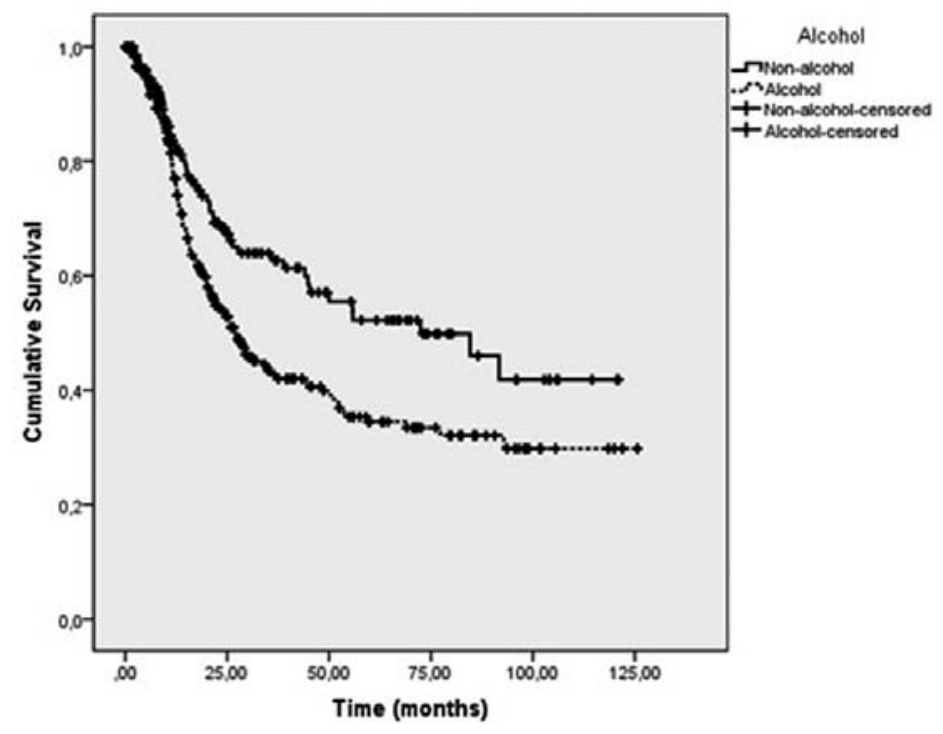

Figura 4. Funciones de sobrevida de pacientes alcoholistas y no alcoholistas.

ganglionares al momento del diagnóstico sea bajo. Según distintos autores el cáncer glótico se presenta en $20 \%$ de los casos con metástasis ganglionares al debut en comparación con $75 \%$ para el cáncer de hipofaringe $^{(11,12)}$.

La sobrevida del cáncer de laringe varía en distintas partes del mundo, indudablemente debido a la diferencia de desarrollo socio-económico-educativo de los distintos países y en función de cómo se comuniquen los re- sultados. Se reportan datos de sobrevida relativa a cinco años muy variables que son del $40 \%{ }^{(13)}, 60,7 \%{ }^{(14)}$, $80 \%{ }^{(15)}$ en la India, Estados Unidos y Japón, respectivamente.

El principal síntoma del cáncer glótico es la disfonía, que, en general, motiva la consulta precoz. El grupo danés de cáncer de cabeza y cuello reporta un hazard ratio para sobrevida global próximo a 1 para estadios precoces al momento del diagnóstico. La captación temprana 
mejora sensiblemente el pronóstico y la sobrevida de estos pacientes ${ }^{(16)}$.

En la actualidad la sobrevida de los pacientes con cáncer de orofaringe está condicionada por la asociación con el virus del papiloma humano (VPH). El estado de VPH positivo confiere un mejor pronóstico para el cáncer de orofaringe, hipofaringe $\mathrm{y}$ adenopatías metastásicas con primitivo desconocido ${ }^{(17,18)}$. El mejor pronóstico se asocia a pacientes de menor edad, no tabaquistas - no alcoholistas, y a una mejor respuesta a la radioquimioterapia $^{(19-21)}$. El estado VPH negativo trae consigo un peor pronóstico, estando asociado a pacientes añosos, con altas tasas de alcoholismo y tabaquismo como en nuestra población ${ }^{(16)}$. La alta densidad de vasos linfáticos en estas regiones anatómicas favorece también la diseminación temprana a ganglios linfáticos regionales. Todavía no contamos con datos nacionales sobre la prevalencia del VPH en los cánceres de cabeza y cuello, pero en nuestra serie los pacientes jóvenes que no presentan factores de riesgo, como el tabaquismo o el alcoholismo, están claramente subrepresentados.

No encontramos diferencia en la sobrevida de aquellos pacientes no tabaquistas comparada con los tabaquistas. El trabajo realizado por López y colaboradores $^{(22)}$ reportó hallazgos similares. De acuerdo con la bibliografía publicada, el consumo de tabaco en pacientes portadores de cáncer de cabeza y cuello disminuye la sobrevida como consecuencia de la mayor incidencia de comorbilidades, principalmente cardiovasculares y respiratorias, así como la presencia de nuevos tumores primarios ${ }^{(23)}$. El uso de tabaco se asocia al aumento de complicaciones posoperatorias hasta seis veces más ${ }^{(24)}$, a lo que se le agrega la mortalidad por patología cardiovascular y respiratoria ${ }^{(25,26)}$.

Nuestros resultados podrían atribuirse al bajo número de no tabaquistas en nuestra población y al alto porcentaje de alcoholistas en este grupo. En segundo lugar, el $48 \%$ de nuestra población no tabaquista se presentó en estadios muy avanzados al momento del diagnóstico, habiéndose asociado un tendencia hacia estadios precoces en otras series de no tabaquistas ${ }^{(27,28)}$.

Por el contrario, encontramos que los pacientes no alcoholistas al momento del diagnóstico presentaron una sobrevida superior a la de los alcoholistas. El metaanálisis conducido por Li y colaboradores ${ }^{(29)}$ demostró que cualquier consumo de alcohol frente al no consumo o consumo ocasional aumenta la mortalidad de pacientes con cáncer de cabeza y cuello. De igual forma se ha demostrado que el consumo de alcohol aumenta las complicaciones postratamiento e incrementa la incidencia de segundos tumores primarios y la mortalidad. El consumo de alcohol está relacionado con la aparición de metástasis ganglionares, factor pronóstico principal en el cáncer de cabeza y cuello ${ }^{(30)}$. Resulta destacable el efecto que tiene el consumo de alcohol sobre el sistema inmune, tanto en lo que respecta a la inmunidad innata como la adaptativa, promoviendo así respuestas pro inflamatorias que conducen al daño tisular en numerosos órganos y al desarrollo de cáncer ${ }^{(31)}$.

Del análisis multivariante (tabla 4) se identifican solo el estadio avanzado y el alcoholismo como factores de mal pronóstico y cuando se estudió la interacción entre alcoholismo y tabaquismo no se observó un efecto sinérgico. Esto último puede estar explicado por el alto porcentaje de estadios avanzados en este subgrupo y, por otro lado, señalar una debilidad de nuestro trabajo que es no tener cuantificado la magnitud del tabaquismo, ya que sabemos que existe una clara asociación dosis respuesta y en el análisis se manejó como una variable dicotómica.

\section{Conclusiones}

La sobrevida del cáncer de cabeza y cuello en Uruguay se encuentra dentro de los rangos publicados mundialmente. En nuestro país persiste como una patología que prevalece en la población masculina mayor de 60 años, con un alto consumo de tabaco y alcohol con respecto a la población general. Se trata de una enfermedad de diagnóstico tardío y por ende presenta una pobre sobrevida, donde el consumo de alcohol parecería incidir negativamente en la sobrevida de estos pacientes. Si bien no se encontramos asociación entre el consumo de tabaco y pronóstico, no debemos dejar de mencionar su rol como principal factor de riesgo asociado al desarrollo de cáncer de cabeza y cuello, principalmente del cáncer de laringe glótico, órgano donde prevalece la enfermedad en nuestro país.

\section{Agradecimiento}

Agradecemos a Guzmán Cuello por el procesamiento de los datos estadísticos.

\section{Abstract}

Introduction: head and neck squamous cell carcinomas (HNSCC) is the most prevalent cancer in otorhinolaryngology, being it the fifth cancer in terms of incidence in Uruguay and the sixth in male mortality. The study aims at analysing global survival in patients with head and neck cancer in two reference centers in Montevideo.

Method: retrospective analysis of global survival of 436 patients diagnosed with HNSCC between 2005 and 2015. Survival was analysed with the Kaplan-Meier method with regard to the patients' stage of cancer, topography, smoking and alcohol consumption. The Cox 
regression method was used to study the interaction of variables with potential importance in the prognosis.

Results: in our series median global survival of patients with head and neck cancer is 35.8 months (23.5-48.1, CI 95\%), it as higher for larynx cancer than that of the rest of the patients with cancer in other anatomic sites, 77.3 (49.4-105.2, CI 95\%) versus 26.2 months (20.7 - 48.1, CI 95\%, p <0,001). There was no difference between the global survival of smokers and non-smokers. Non-alcohol consumers presented a better global survival than the group of alcohol consumers, 72.4 (39.1-105.7, CI 95\%) versus 26.7 (19.9-33.6 CI 95\%). The multivariable analysis identified stage of cancer and alcohol consumption as independent prognostic factors in our series.

Conclusions: median global survival in our series is similar to that reported in the international bibliography. Patients with larynx cancer evidence a longer survival than patients with cancer in other otorhinolaryngologic sites. As to classic risk factors, alcohol consumption has a negative impact on the survival of patients with head and neck cancer.

\section{Resumo}

Introdução: o carcinoma epidermóide de cabeça e pescoço (HNSCC) é o câncer com maior prevalência em Otorrinolaringologia; no Uruguai é o quinto tumor em incidência e o sexto em mortalidade em homens. $\mathrm{O}$ objetivo deste estudo foi analisar a sobrevida global dos pacientes com câncer de cabeça e pescoço em dois centros de referência de Montevidéu.

Métodos: fez-se uma análise retrospectiva da sobrevida global de 436 pacientes diagnosticados com HNSCC no período 2005-2015. A sobrevida foi estudada pelo método de Kaplan-Meier em relação ao estádio tumoral, topografia, estatus de tabaquismo e alcoolismo dos pacientes. Para estudar a interação das variáveis com possível importância no prognóstico utilizou-se o modelo de regressão de Cox.

Resultados: a mediana de sobrevida global dos pacientes com câncer de cabeça e pescoço na nossa serie foi de 35,8 meses (23,5-48,1, IC 95\%), sendo superior para o câncer de laringe ao do resto dos pacientes com câncer de outros sítios anatômicos, 77,3 (49,3-105,2, IC $95 \%$ ) versus 26,2 meses $(20,7-48,1$, IC 95\%, p < 0,001). Não se observou diferença entre a sobrevida global de fumantes e não fumantes. $\mathrm{O}$ grupo dos não alcoolistas apresentou uma melhor sobrevida global que o grupo dos alcoolistas, 72,4 (39,1-105,7, IC 95\%) versus 26,7 (19,9-33,6 IC 95\%). A análise multivariante identificou o estádio e o alcoolismo como fatores prognósticos independentes na nossa serie.
Conclusões: a mediana de sobrevida global da nossa serie é similar à descrita na bibliografia internacional. Os pacientes com câncer de laringe apresentam maior sobrevida que os pacientes com câncer de outros sítios otorrinolaringológicos. Dos fatores de risco clássicos, na nossa serie o consumo de álcool impacta negativamente na sobrevida dos pacientes com câncer de cabeça e pescoço.

\section{Bibliografía}

1. Ferlay J, Soerjomataram I, Ervik M, Dikshit R, Eser S, Mathers C, et al. GLOBOCAN 2012 v1 .0, Cancer Incidence and Mortality Worldwide: IARC Cancer Base No. 11. Lyon, France: International Agency for Research on Cancer, 2013. Disponible en: http://globocan.iarc.fr. [Consulta: 7 febrero 2017].

2. Warnakulasuriya S. Global epidemiology of oral and oropharyngeal cancer. Oral Oncol 2009; 45(4-5):309-16.

3. Barrios E, Garau M, Alonso R, Musseti C. IV Atlas de Incidencia del Cancer: período 2007-2011. Montevideo: Registro Nacional del Cancer, Comision Honoraria de Lucha Contra el Cancer, 2014. Disponible en: http://www.comisioncancer.org.uy/uc_394_1.html [Consulta: 23 octubre 2016].

4. Perdomo S, Martin Roa G, Brennan P, Forman D, Sierra MS. Head and neck cancer burden and preventive measures in Central and South America. Cancer Epidemiol 2016; 44(Suppl 1):S43-52.

5. Quer Agusti M, López Vila M. Características clínicas de los tumores de laringe. En: Suárez Nieto C, Gil-Carcedo García LM, Algarra JM, Medina JE, Ortega del Álamo P, Trinidad Pinedo J. Tratado de otorrinolaringologia y cirugía de cabeza y cuello: tomo 4. 2 ed. Buenos Aires: Médica Panamericana; 2008:3515-28.

6. Global Adult Tobacco Survey. Global Adult Tobacco Survey: fact sheet Uruguay 2009. Geneva: WHO, 2010. Disponible en: http://www.who.int/tobacco/surveillance/

fact_sheet_of_gats_uruguay_2010.pdf. [Consulta: 7 febrero 2017].

7. Boado M, Bianco E. Primera encuesta nacional de tabaquismo: Uurguay 2008. Tend Med 2010; 36(5):26-31.

8. Shah JP, Patel SG, Singh B. Jatin Shah's head and neck: surgery and oncology. 4 ed. Philadelphia, PA: Elsevier; 2012.

9. Chung EJ, Kim GW, Cho BK, Park HS, Rho YS. Pattern of lymph node metastasis in hypopharyngeal squamous cell carcinoma and indications for level VI lymph node dissection. Head Neck 2016; 38(Suppl 1):E1969-73.

10. Papadas TA, Alexopoulos EC, Mallis A, Jelastopulu E, Mastronikolis NS, Goumas P. Survival after laryngectomy: a review of 133 patients with laryngeal carcinoma. Eur Arch Otorhinolaryngol 2010; 267(7):1095-101.

11. Pinilla M, Gónzalez FM, López-cortijo C, Vicente J, Górriz C, Laguna D, et al. Afectación ganglionar cervical en el carcinoma laríngeo: un estudio retrospectivo de 430 casos. Acta Otorrinolaringol Esp 2001; 52(3):213-8. 
12. Gallo O, Boddi V, Bottai GV, Franchi A, Storchi OF. Prognostic significance of clinically false positive cervical lymph nodes in patients with laryngeal carcinoma. Cancer 1995; 75(5):1066-83.

13. Yeole B, Sankaranarayanan R, Sunny L, Sawaminathan R, Parkin D. Survival from head and neck cancer in Mumbai (Bombay), India. Cancer 2000; 89(2):437-4.

14. National Cancer Institute: Surveillance, Epidemiology and Ends Results Programme. Cancer stat facts: laryngeal cancer 2007-2014. Bethesda, MD: NCI, 2014. Disponible en: https://seer.cancer.gov/statfacts/html/laryn.html. [Consultado: 5 mayo 2017].

15. Machii R, Saika K. Five-year relative survival rate of larynx cancer in the USA, Europe and Japan. Jpn J Clin Oncol 2014; 44(10):1015-6.

16. Lyhne NM, Johansen J, Kristensen C, Andersen E, Primdahl H, Andersen L, et al. Incidence of and survival after glottic squamous cell carcinoma in Denmark from 1971 to 2011: a report from the Danish Head and Neck Cancer Group. Eur J Cancer 2016; 59:46-56.

17. Lai K, Killingsworth M, Matthews S, Caixeiro N, Evangelista $\mathbf{C}, \mathbf{W u} \mathbf{X}$, et al. Differences in survival outcome between oropharyngeal and oral cavity squamous cell carcinoma in relation to HPV Status. J Oral Pathol Med 2017; 46(8):574-82.

18. Sivars L, Tani E, Näsman A, Ramqvist T, Munck-Wikland E, Dalianis T. Human papillomavirus as a diagnostic and prognostic tool in cancer of unknown primary in the head and neck region. Anticancer Res 2016; 36(2):487-94.

19. Dayyani F, Etzel CJ, Liu M, Ho C-H, Lippman SM, Tsao AS. Meta-analysis of the impact of human papillomavirus (HPV) on cancer risk and overall survival in head and neck squamous cell carcinomas (HNSCC). Head Neck Oncol 2010; 2:15.

20. Curado MP, Boyle P. Epidemiology of head and neck squamous cell carcinoma not related to tobacco or alcohol. Curr Opin Oncol 2013; 25(3):229-34.

21. Hong AM, Dobbins TA, Lee CS, Jones D, Harnett GB, Armstrong BK, et al. Human papillomavirus predicts outcome in oropharyngeal cancer in patients treated primarily with surgery or radiation therapy. $\mathrm{Br} \quad \mathrm{J}$ Cancer 2010; 103(10):1510-7.

22. Lopez R, Zago M, Eluf-Neto J, Curado MP, Daudt AW, da Silva-Junior WA, et al. Education, tobacco smoking, alcohol consumption, and IL-2 and Il-6 gene polymorphisms in the survival of head and neck cancer. Braz J Med Biol Res 2011; 44(11):1006-12.

23. Wald NJ, Hackshaw AK. Cigarette smoking: an epidemiological overview. Br Med Bull 1996; 52(1):3-11.

24. van Imhoff L, Kranenburg G, Macco S, Nijman N, van Overbeeke E, Wegner I, et al. Prognostic value of continued smoking on survival and recurrence rates in patients with head and neck cancer: a systematic review. Head Neck 2016; 38 (1):14-20.

25. Hatcher JL, Sterba KR, Tooze JA, Day TA, Carpenter MJ, Alberg AJ, et al. Tobacco use and surgical outcomes in patients with head and neck cancer. Head Neck 2016; 38(5):700-6.

26. Sharp L, McDevitt J, Carsin AE, Brown C, Comber H. Smoking at diagnosis is an independent prognostic factor for cancer-specific survival in head and neck cancer: findings from a large, population-based study. Cancer Epidemiol Biomarkers Prev 2014; 23(11):2579-90.

27. Dahlstrom KR, Little JA, Zafereo ME, Lung M, Wei Q, Sturgis EM. Squamous cell carcinoma of the head and neck in never smoker-never drinkers: a descriptive epidemiologic study. Head Neck 2008; 30(1):75-84.

28. Durr ML, Li D, Wang SJ. Oral cavity squamous cell carcinoma in never smokers: analysis of clinicopathologic characteristics and survival. Am J Otolaryngol 2013; 34(5):388-93.

29. Li Y, Mao Y, Zhang Y, Cai S, Chen G, Ding Y, et al. Alcohol drinking and upper aerodigestive tract cancer mortality: A systematic review and meta-analysis. Oral Oncol 2014; 50(4):269-75.

30. Moyses RA, López RVM, Cury PM, Siqueira SAC, Curioni OA, de Gois Filho JF, et al. Significant differences in demographic, clinical, and pathological features in relation to smoking and alcohol consumption among 1,633 head and neck cancer patients. Clinics (Sao Paulo) 2013; 68(6):738-44.

31. Szabo G, Saha B. Alcohol's effect on host defense. Alcohol Res 2015; 37(2):159-70. 